\title{
Polarized Radiative Transfer Equation in Some Geometries of Elliptic Type
}

\author{
Juris Freimanis ${ }^{1,2}$ \\ ${ }^{1}$ Ventspils International Radio Astronomy Centre, Ventspils University College, \\ Inzenieru iela 101a, LV-3600 Ventspils, Latvia \\ email: jurisf@venta.lv \\ ${ }^{2}$ Institute of Mathematical Sciences and Information Technologies, Liepaja University, \\ Liela iela 14, LV-3401 Liepaja, Latvia
}

\begin{abstract}
A general method, which allows us to derive explicit expressions for the differential operator of stationary quasi-monochromatic polarized radiative transfer equation in Euclidean space, with piecewise homogeneous real part of the effective refractive index, is applied to ellipsoidal, oblate spheroidal, prolate spheroidal and elliptic conical coordinate systems.
\end{abstract}

Keywords. Polarization, radiative transfer, methods: analytical

\section{Introduction}

While modeling polarized radiative transfer in stellar and planetary atmospheres, circumstellar disks with exoplanets orbiting inside them, close binary systems etc., generally the radiative transfer equation (RTE) should be written and solved in such a coordinate system which reflects the symmetry of the physical problem - at least the symmetry of matter distribution; the symmetry of radiation field is usually somewhat lower.

Let us consider a homogeneous isotropic host medium in Euclidean space, with polydisperse scatterers (e.g. circumstellar dust) of volume concentration, $n_{0}$. The conditions of validity of stationary quasi-monochromatic RTE (Mishchenko et al. 2006, paragraphs 8.11 and 8.15; Mishchenko 2008a, Mishchenko 2008b) are satisfied, with possible addition of internal primary radiation sources as in Freimanis (2011). The effective refractive index (host medium with scatterers) is only weakly anisotropic so that birefringence can be neglected. The real part of the effective refractive index is piecewise homogeneous; radiation propagates along straight lines in each homogeneous part of the space.

We denote the Stokes 4 -vector in the point of observation, $\mathbf{r}$, by $\mathbf{I}(\mathbf{r}, \vartheta, \varphi)$, where the spherical angles $(\vartheta, \varphi)$ describe the direction of propagation with respect to spatial basis vectors, the imaginary part of the wavenumber in the host medium by $k^{\prime \prime}$, the statistically averaged particle extinction matrix by $\mathbf{K}(\vartheta, \varphi)$, the statistically averaged particle phase matrix by $\mathbf{Z}\left(\vartheta, \varphi ; \vartheta^{\prime}, \varphi^{\prime}\right)$, and the primary source function by $\mathbf{I}_{0}(\mathbf{r}, \vartheta, \varphi)$. The polarized RTE is as follows (see Mishchenko et al. 2006, Mishchenko 2008b, Freimanis 2011):

$$
\begin{aligned}
\frac{d \mathbf{I}(\mathbf{r}, \vartheta, \varphi)}{d s} & -\mathbf{U}_{1} \mathbf{I}(\mathbf{r}, \vartheta, \varphi) \frac{d \psi}{d s} \\
& =-k^{\prime \prime} \mathbf{I}(\mathbf{r}, \vartheta, \varphi)-n_{0} \mathbf{K}(\vartheta, \varphi) \mathbf{I}(\mathbf{r}, \vartheta, \varphi) \\
& +n_{0} \int_{4 \pi} \mathbf{Z}\left(\vartheta, \varphi ; \vartheta^{\prime}, \varphi^{\prime}\right) \mathbf{I}\left(\mathbf{r}, \vartheta^{\prime}, \varphi^{\prime}\right) \sin \vartheta^{\prime} d \vartheta^{\prime} d \varphi^{\prime}+\mathbf{I}_{0}(\mathbf{r}, \vartheta, \varphi),
\end{aligned}
$$

where $d \mathbf{I} / d s$ is the derivative of the Stokes vector along the path of propagation, $d \psi / d s$ is the speed of rotation of polarization reference basis vectors around the direction of propagation, and $\mathbf{U}_{1}$ is the transformation matrix of Stokes vector upon infinitesimal 
rotation of the reference system. It is assumed that the polarization reference plane is that going through the spatially variable polar axis $\vartheta=0$ and the direction of propagation of radiation; as a result, in curvilinear coordinate system this plane generally rotates.

The left-hand side of Eq. (1.1) is a differential operator with special expression in each particular coordinate system; the right-hand side is one and the same in all coordinate systems. The aim of this study is to find explicit expressions for the differential operator of RTE in some coordinate systems of elliptic type mentioned in Korn \& Korn (1968), applying the general method developed in Freimanis (2011).

\section{Summary of the results}

Standard procedures described in Freimanis (2011) were applied to the following orthogonal coordinate systems:

(a) Ellipsoidal system with the longest axis of ellipsoids coinciding with Descartes' $x$ axis, and the shortest axis coinciding with Descartes' $z$ axis. An alternative parameterization of the ellipsoidal coordinates was introduced;

(b) Standard oblate spheroidal system (Korn \& Korn 1968), as a particular case of the system mentioned above;

(c) Standard prolate spheroidal system (Korn \& Korn 1968);

(d) Elliptic conical system, with alternative parameterization.

Clear expressions for the differential operator of polarized RTE in all these cases were found. The respective formulae are very long in the case of a triaxial ellipsoidal system but only moderately long in the other cases. Besides, alternative parameterization for an ellipdoidal coordinate system with the longest axis of ellipsoids coinciding with Descartes' $z$ axis, and the shortest axis coinciding with Descartes' $y$ axis is offered as well. (The only important feature for the offered redefinitions of ellipsoidal coordinate systems is that the $z$ axis must be the longest or the shortest; the $x$ and $y$ axis can be mutually exchanged. The given redefinition is not valid if the $z$ axis coincides with the middle axis of ellipsoids.)

\section{Acknowledgements}

This study was financed from the basic budget of Ventspils International Radio Astronomy Centre, as well as from co-sponsorship by Ventspils City Council and from Latvian Council of Science grant No. 11.1856. Some financing was provided by the Institute of Mathematical Sciences and Information Technologies of Liepaja University as well. The participation of the author at the Symposium was financed from European Regional Development Fund's project SATTEH (2010/0189/2DP/2.1.1.2.0/10/APIA/VIAA/019) being implemented in Ventspils University College. The author expresses his gratitude to all these entities.

\section{References}

Mishchenko, M. I., Travis, L. D., \& Lacis, A. A. 2006, Multiple Scattering of Light by Particles. Radiative Transfer and Coherent Backscattering. (Cambridge et al.: Cambridge University Press)

Mishchenko, M. I. 2008a, Optics Express, 16, 2288

Mishchenko, M. I. 2008b, Journal of Quantitative Spectroscopy and Radiative Transfer, 109, 2386

Freimanis, J. 2011, Journal of Quantitative Spectroscopy and Radiative Transfer, 212, 2134

Korn, G. A. \& Korn, T. M. 1968, Mathematical Handbook for Scientists and Engineers. (New York et al:: McGraw-Hill Book Company) 